\title{
Transfer Function of A Crossed Wire Pendulum Isolation System
}

Nobuyuki Kanda, Mark A. Barton, and Kazuaki Kuroda

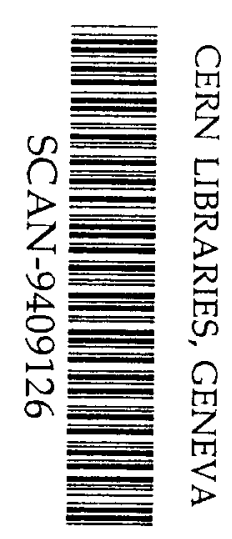

( July, 1994)

Submitted to Review of Scientific Instruments 



\title{
Transfer Function of A Crossed Wire Pendulum Isolation System
}

\author{
Nobuyuki Kanda, Mark A. Barton, and Kazuaki Kuroda \\ Institute for Cosmic Ray Research, University of Tokyo 3-2-1, Midoricho, Tanashi, Tokyo 188
}

(July 16, 1994)

\begin{abstract}
We measured the vibrational transfer function of a newly proposed crossed wire pendulum in the frequency range 0.03 to $3 \mathrm{~Hz}$. We confirmed that it behaves as a much longer simple pendulum of the same period as far as one dimensional movement of its center of mass is concerned. We conclude that the pendulum shows promise as a component in an ultra-low frequency antivibration system.
\end{abstract}

\section{INTRODUCTION}

A crossed wire pendulum (X-pendulum) with a period of several tens of seconds has been proposed [1] for such uses as an ultra-low frequency vibration isolation system (equivalent to a very long simple pendulum) or a suspension for accelerometers in precision measurement. In particular it is a candidate for use in the seismic isolation stage of a long baseline laser interferometer to detect gravitational waves from astronomical sources. The existing isolation system in such interferometers contains a series of mechanical low-pass filters (simple harmonic oscillators), the final stage being a simple pendulum with a period of about $1 \mathrm{~s}$ [2] [3]. Since low frequency seismic noise leaking through the anti-vibration system is the limiting factor for the ultimate noise in the laser interferometer at the low end of the spectrum, lowering the resonant frequency of each stage in the isolation system much enhances its reduction ratio and reduces the load of the local control system for the mirror pendulum [4].

The proposed X-pendulum with its longer period has the potential to improve the reduction ratio in the low frequency range. Since this $\mathrm{X}$-pendulum is a passive pendulum, not an active anti-vibration system adopting feedback techniques, it not only has extremely low resonant frequency but also shows promise for use in gravity wave detectors for its simplicity, durability, and stability. It is the main object of this paper to prove that this system behaves as a long period simple pendulum in one dimension, i.e., as a mechanical low pass filter.

This paper presents the first experimental result for the transfer function of this $\mathrm{X}$ pendulum from 0.03 to $3 \mathrm{~Hz}$. Typically the period was set around a few seconds due to limited measuring time. 


\section{TRANSFER FUNCTION OF THE CROSSED WIRE PENDULUM}

The X-pendulum used in the present experiments is shown schematically in Fig. 1. The angular frequency of the principal resonance, $\omega_{0}^{2}$, is given by the equation [1]

$$
\omega_{0}^{2}=\frac{M g\left(H-H_{H}\right)}{M(L \tan \alpha / 2+H)^{2}+I_{0}}
$$

where

$$
H_{H}=L(\cot \alpha-\tan \alpha) / 4
$$

where $g$ is the gravitational acceleration, $\ell$ is the wire length, $L$ is the distance between either the top or the bottom suspension points, $M$, and $I_{0}$ are respectively the mass and the moment of inertia of the moving part, and $\alpha$ is the angle shown in Fig. 1. The position of the center of mass, $H$, and the critical position, $H_{H}$, are measured downwards from the lower plate. Typically $H$ is greater than, i.e., below $H_{H}$. As $H$ approaches $H_{H} \omega_{0}$ becomes zero (infinite period).

The transfer function of the system measured at the center of mass falls off with frequency according to

$$
\begin{aligned}
T(\omega) & =x_{\text {out }} / x_{i n}=\frac{-r \omega^{2}+\omega_{0}^{2}(1+i \phi)}{\omega_{0}^{2}-\omega^{2}+i \phi \omega_{0}^{2}} \\
& =T_{0}(\omega)+\frac{-r \omega^{2}}{\omega_{0}^{2}-\omega^{2}+i \phi \omega_{0}^{2}}
\end{aligned}
$$

with

$$
T_{0}(\omega)=\frac{\omega_{0}^{2}(1+i \phi)}{\omega_{0}^{2}-\omega^{2}+i \phi \omega_{0}^{2}}
$$

and

$$
r=\frac{I_{0}}{M l_{m a s s}^{2}+I_{0}}
$$

where $\omega_{0}$ is the angular resonant frequency, $M$ is the mass of the structure, $l_{\text {mass }}$ is the length from the crossing points of wires to the center of mass, and $I_{0}$ is the moment of inertia of the structure around the center of mass. $\phi$ represents the fractional imaginary component of the restoring force of the pendulum as in the usual extension to Hooke's law [5] and is assumed to be frequency independent. $T_{0}(\omega)$ is the transfer function of a simple pendulum with a massless (but not frictionless) suspension wire. In the range $\omega \ll \omega_{0}$, the seismic noise passes directly to the mass, whereas in the range $\omega \gg \omega_{0}$ the noise is proportional to the inverse square of $\omega$.

In our experiment, there are two factors which make the transfer function of this $\mathrm{X}$ pendulum different from a simple pendulum. One, the moment of inertia of the structure, has already been considered in (3). Notwithstanding the smallness of $r(2.8 \%$ for the setup 
in Fig. 1), the effect at higher frequencies cannot be negligible. The second is the error in positioning the position sensor directly opposite the center of mass. Note that although the center of mass behaves as if it were hung as a long simple pendulum of the same period, the attitude of the hung mass changes as for a much shorter pendulum of the same length. If we measure a point which is displaced slightly from the center of mass, the rotation angle of the structure appears in the transfer function as follows:

$$
T^{\prime}(\omega)=T(\omega)+b
$$

where $b$ represents direct coupling between $x_{\text {in }}$ and $x_{\text {out }}$. The sign of $b$ is positive when the measured point is above the center of mass, or negative when below. The transfer function $T^{\prime}(\omega)$ is given by

$$
\begin{gathered}
T^{\prime}(\omega)=\frac{-(r+b) \omega^{2}+(1+b) \omega_{0}^{2}(1+i \phi)}{\omega_{0}^{2}-\omega^{2}+i \phi \omega_{0}^{2}} \\
=T_{0}(\omega)+\frac{-(r+b) \omega^{2}}{\omega_{0}^{2}-\omega^{2}+i \phi \omega_{0}^{2}}+\frac{b \omega_{0}^{2}(1+i \phi)}{\omega_{0}^{2}-\omega^{2}+i \phi \omega_{0}^{2}}
\end{gathered}
$$

The squared magnitude of the transfer function $T^{\prime}(\omega) T^{\prime *}(\omega)$ is

$$
T^{\prime}(\omega) T^{\prime *}(\omega)=|T(\omega)|^{2}+b^{2}+2 b \Re[T(\omega)]
$$

Fig. 2 shows $T^{\prime}(\omega) T^{\prime *}(\omega)$ with $b=-0.01,-0.03,0,0.01,0.03$, assuming $r=0$, and with $b=0$, assuming $r=0.02$. The noise suppression is limited to $-30 \mathrm{~dB}$ with $b=0.03$. Moreover in the case of negative $b$, the transfer function has a dip. If we choose $b=-r$ ( $r$ is always positive), the term in $r$ vanishes and the transfer function can be represented as

$$
T^{\prime}(\omega ; b=-r)=(1+b) T_{0}(\omega)
$$

Hence, it is important to tune the sensor position for a precise measurement of the transfer function. We show the measurement of $T^{\prime}(\omega)$ with typical $b$ values in following section.

The damping due to the wires in an $\mathrm{X}$-pendulum is given as the quality factor $Q$ in terms of the mass position $H[1]$ as follows:

$$
Q=\frac{\frac{H}{H_{H}}-1}{\frac{2 \phi_{W}}{H_{H}}\left(\frac{E_{W} C_{W}}{M g \sin \alpha_{0}}\right)^{1 / 2}}
$$

where $\phi_{W}$ is the fractional imaginary component of the elasticity of the wire material representing the intrinsic damping. $E_{W}$ and $C_{W}$ are the Young's modulus and the moment of area of the wire, respectively. Argument for the frequency dependence of $\phi_{W}$ is seen in several papers by Saulson et al [5]. 


\section{EXPERIMENTAL SETUP}

As can be seen in Fig. 1, the top plate to which the four wires were connected was fixed under a one-dimensional sliding mechanism and was excited by an electromagnetic actuator in the horizontal direction. We could easily adjust the pendulum resonant frequency by tuning $H$. The top plate was excited by white noise from a spectrum analyzer with a maximum amplitude of about $0.1 \mathrm{~mm}$ (limited so as not to let the output of the mass displacement signal enter the nonlinear region of the photo sensor used). The pendulum was set on a firm base made by stacking lead blocks on the experimental room floor.

To measure the transfer function of this system, we needed to measure the displacement of the supporting plate and that of the center of mass of the pendulum. As noted above the angular displacement of the mass is a potential problem. Therefore ideally, the sensor of the mass position should not be sensitive to the angular displacement, but it is not easy to use such a sensor for this measurement. Since we are particularly interested in the behavior as an anti-vibration system, we considered the frequency range from 0.03 to $3 \mathrm{~Hz}$. In this range, the expected seismic noise varies from $110 \mu \mathrm{m} / \sqrt{ } \mathrm{Hz}$ at $0.03 \mathrm{~Hz}$ to $110 \mathrm{~nm} / \sqrt{ } \mathrm{Hz}$ at $3 \mathrm{~Hz}$ at our campus in a suburb of Tokyo. This means that the displacement sensor needed a wide dynamic range. We used two photo-sensors each of which consisted of a light-emitting diode and a photosensitive diode pair for measuring displacements of the top plate and the center of the suspended mass. Fig. 3 shows the output of the sensor as a function of displacement. The linear region defining the effective dynamic range is indicated. The electric noise of this sensor is about $800 \mu \mathrm{V} / \sqrt{\mathrm{Hz}}$ at $1 \mathrm{~Hz}$, which corresponds to displacement noise of $0.5 \mu \mathrm{m} / \sqrt{ } \mathrm{Hz}$. Both sensors were calibrated using a dial gauge, to an accuracy of $10 \mu \mathrm{m}$. Before the measurement, we checked electric cross talk between the two sensors and that due to the electric actuator and confirmed they it were less than the electrical noise of the sensors. The spectra obtained from the sensors were recorded and used to calculate the transfer function of the pendulum. The sensor near the center of mass was placed on a small stage which could be finely adjusted by a micrometer head.

Besides confirming that the crossed pendulum was equivalent to the simple one, we also measured the loss of the wires. Keeping the angle of the crossed wires unchanged we could not easily change the intrinsic loss factor of the wire without substituting wire of different diameter or different material. Instead, we looked for a change of $Q$ with change of $H$ and hence of the resonant frequency. We used $\phi 100 \mu \mathrm{m}$ piano wire supplied by Nilaco Corporation and set the resonant frequency to three different values, $0.38 \mathrm{~Hz}, 0.29 \mathrm{~Hz}$ and $0.21 \mathrm{~Hz}$.

Finally we checked the change in the transfer function due to simulated errors in aligning the sensor with the center of mass of the pendulum. The resonant frequency used in this stage of the experiment was $0.23 \mathrm{~Hz}$.

\section{RESULTS AND DISCUSSION}

Fig. 4 shows the transfer function for three different values of the direct coupling $b$ at the same resonant frequency. First the mass position with $b^{\prime}=b-r$ was pinpointed. The distance from the point of $b^{\prime}=0$ to the calculated center of mass was approximately $3.5 \mathrm{~mm}$. This corresponds to $b=-0.03$, which agrees quite well with the calculated value of $r, 0.028$. 
Then, using a micrometer head, the mass position was adjusted relative to this origin to give successively $b^{\prime}=-0.03$ and $b^{\prime}=0.03$. The solid line shows the transfer function $T_{0}(\omega) T_{0}^{*}(\omega)$ in (5). The response of the $\mathrm{X}$-pendulum conforms well to the model.

Fig. 5 represents the squared amplitude of the transfer function of the pendulum for three different resonant frequencies, $0.38 \mathrm{~Hz}, 0.29 \mathrm{~Hz}$, and $0.21 \mathrm{~Hz}$. Although phase information has been surpressed in Fig. 5, we confirmed from the complex values reported by the frequency analyzer that the phase changes to $\pi$ at resonance in accordance with Bode's law. The overall structure of this response is very similar to that of a ideal simple pendulum except for two features. One is that we have a second harmonic of the resonance in each case due to the nonlinearity of the sensor (Fig. 3). The other is that there is a small resonant peak around $2-3 \mathrm{~Hz}$ due to the lateral motion of the pendulum (which would not appear if the sensor detected only pure one dimensional displacement). The frequency of this second lateral resonance can be shifted higher by making wider the distance between the two pairs of wires and the loss factor of the mode can be reduced by adopting thick wires.

To examine $Q$, three runs were taken with different resonant frequencies, with $b^{\prime}$ tuned to zero in each case. The loss factor $\phi$ of the pendulum was obtained by curve fitting using the squared modulus of (5). The $Q$ of the pendulum is then $1 / \phi$. Table I shows the values of $Q$ obtained, together with the implied values of the intrinsic loss factor of the wire, $\phi_{W}$ from (12). The following parameter values were assumed: $E_{W}=2.07 \times 10^{11} \mathrm{~Pa}$, $A_{W}=7.85 \times 10^{-9} \mathrm{~m}^{2}, H=0.1 \mathrm{~m}, g=9.8 \mathrm{~ms}^{-2}$, and $\alpha=0.197$. The factor $H / H_{H}-1$ in the numerator represents the factor by which the square of the period has been reduced relative to a simple pendulum of equal length, as can easily be seen by comparing with (1). This fact was used in conjunction with the measured periods to determine $H / H_{H}-1$. Damping from flexure of the rigid superstructure of the pendulum, and air resistance were estimated and found to be negligible. However the values of $\phi_{W}$ obtained are too large to be realistic and suggest a unidentified source of damping.

In this experiment we proved that the X-pendulum works as a low pass mechanical filter as far as its center of mass is concerned, if we can neglect the moment of inertia of the suspended mass and supporting components. Since the single X-pendulum is inevitably accompanied by the rotation of the suspension frame, a new design for evading this is now under consideration and will be disclosed elsewhere. It is not difficult to set the resonant frequency lower than those in this measurement. Since as pointed in Ref. [1], $20 \mathrm{~s}$ period was easily obtained with a somewhat larger device and $100 \mathrm{~s}$ is possibly achievable, this result confirmed that this X-pendulum can be used to develop an anti-vibration system working in the very low frequency region. Especially in laser interferometers for gravity wave detectors, since reducing horizontal vibration is the first priority, including one or more stages of this type of pendulum will allow the frequency range to be extended downwards. 


\section{REFERENCES}

[1] M. A. Barton and K. Kuroda, submitted to Rev.Sci.Instrum.

[2] A. Abramovici et al., Science 256, 325 (1992).

[3] C. Bradashia et al., Nucl. Instrum. Methods Phys. Res. A289, 518 (1990).

[4] S. Kawamura and M. E. Zucker, Appl. Opt. 33, 3912-3918 (1994).

[5] P. R. Saulson, Phys. Rev. D42, 2437 (1990).

[6] P. R. Saulson et al., Rev. Sci. Instrum. 65, 182 (1994), and references therein.

\section{FIGURE CAPTIONS}

Fig. 1. The $\mathrm{X}$-pendulum shown in side view with its driving mechanism.

The upper of the top plates was fixed under a horizontal linear sliding mechanism. The lower plate firmly held the rigid stem of the mass. These top plates were connected by two pairs of wires. In each pair, the wires formed the shape of a X, approximately in the same plane but slightly displaced so as not to touch each other. The upper photo-sensor measured the motion of the slider and the lower photo-sensor measured the horizontal movement of the mass.

Fig. 2. Squared magnitude of the transfer function.

Squared magnitude of the transfer function $T^{\prime}(\omega) T^{\prime *}(\omega)$ is affected both by a direct coupling $b$ between $x_{i n}$ and $x_{\text {out }}$ through the first order rotation of the rigid frame and by an effect $r$ of the moment of inertia of the frame. The two effects combine almost linearly. $T^{\prime} T^{\prime *}$ is shown as a function of $f=\frac{\omega}{2 \pi}$. Solid line: $b=0$, dashed line: $b= \pm 0.01$, long-dashed line: $b= \pm 0.03$ with $r=0$ and dotted line: $r=0.02$ with $b=0$.

Fig. 3. Calibration of Photo Sensor.

The sensitivity curve of the photo-sensor used to detect the displacement. The sensor consisted of a light-emitting diode and a photo-sensitive diode pair.

Fig. 4. Measurements and curve fitting to obtain the loss factor of the pendulum.

The figure shows the measurements of the squared magnitude transfer function with various values of $b^{\prime}=b-r$. The offsets of the sensor from the point $b^{\prime}=0$ are $0 \mathrm{~mm}$ for circles, $+3.5 \mathrm{~mm}$ for ' $x$ ' symbols, and $-3.5 \mathrm{~mm}$ for ' + ' symbols. These correspond to increments in $b$ of 0.03 . The solid line was obtained by curve-fitting on the circle data points using the squared modulus of (5), with $\phi$ and $\omega_{0}$ as free parameters. Agreement is fairly good.

Fig. 5. Transfer functions for three different resonant frequencies.

The overall structure of the response is very similar to that of an ideal simple pendulum. See text for detail. 


\section{FIGURES}

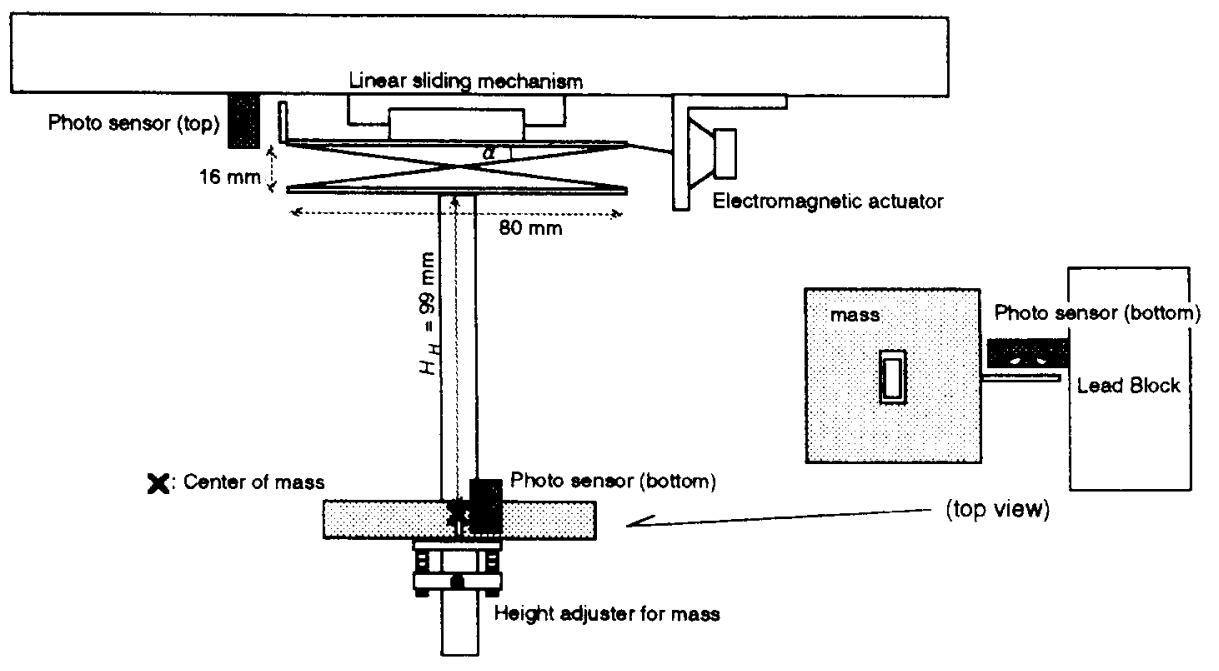

FIG. 1.

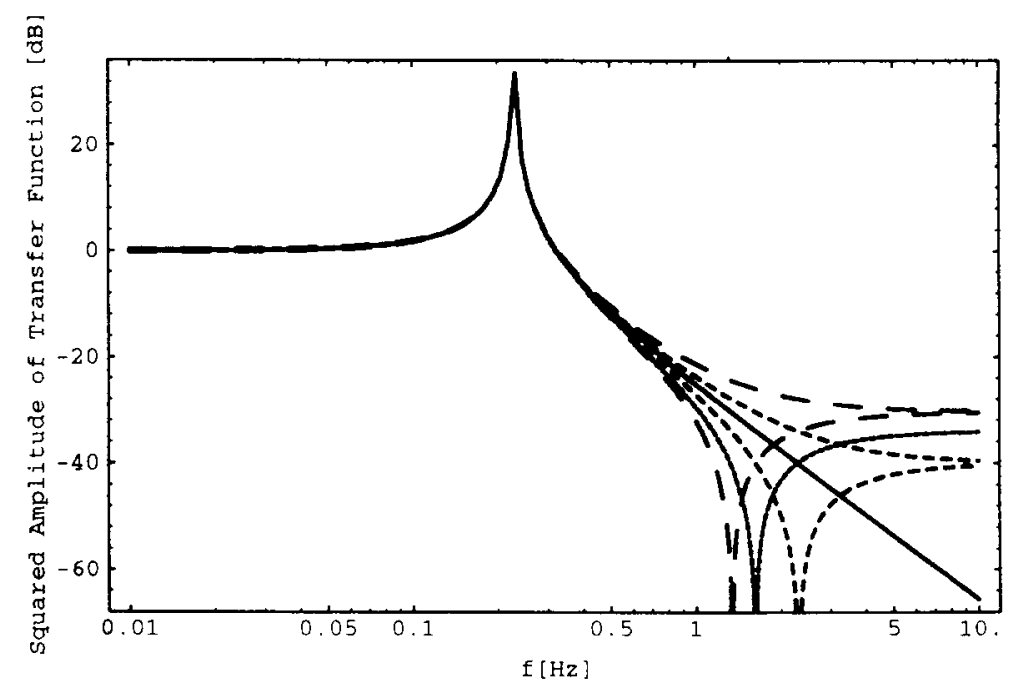

FIG. 2. 


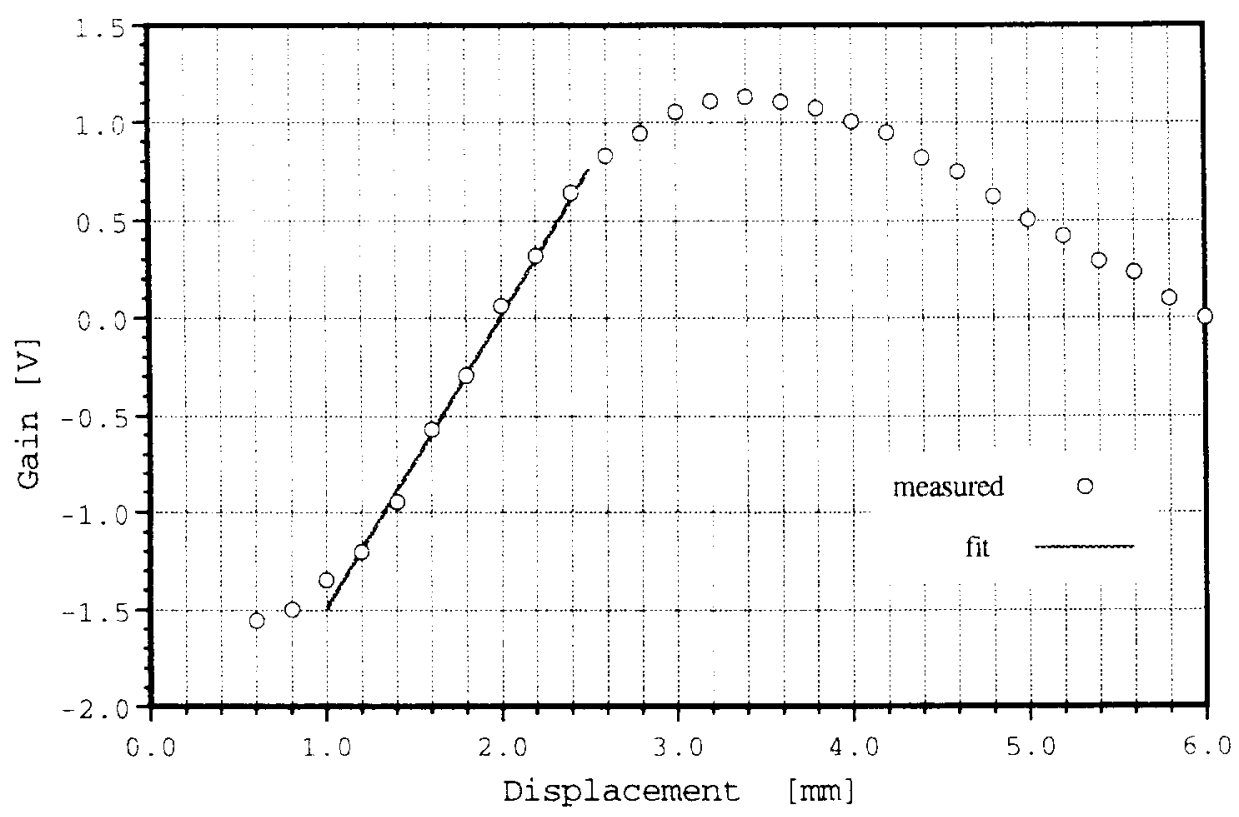

FIG. 3.

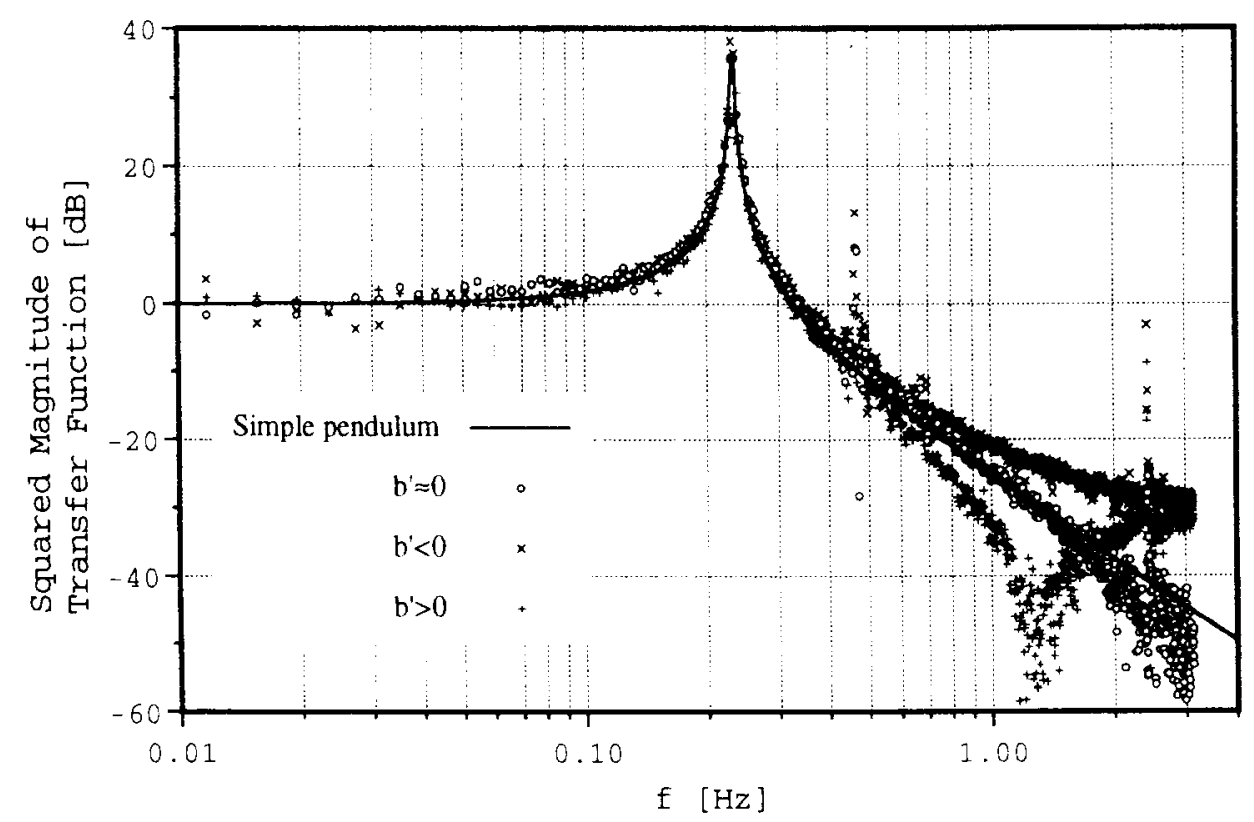

FIG. 4. 


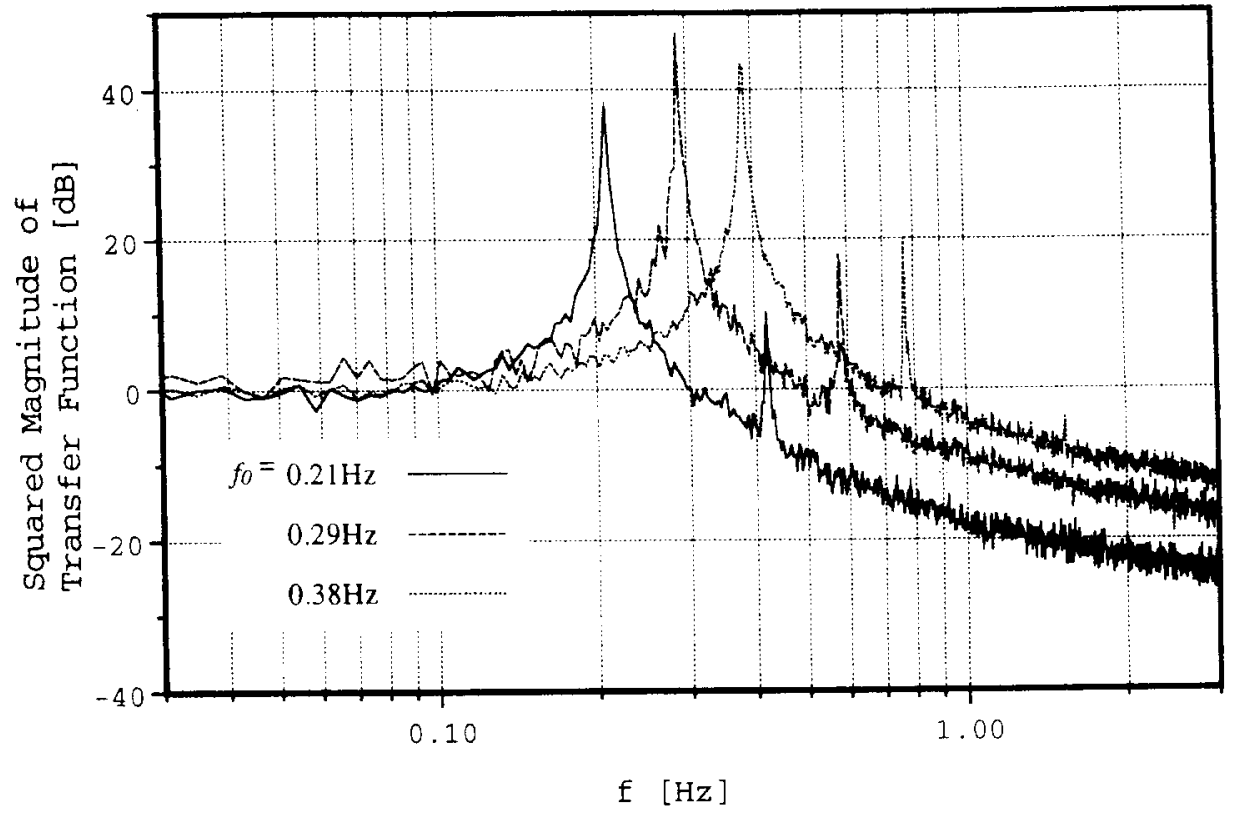

FIG. 5 . 


\section{TABLES}

TABLE I. The change in $Q$ with resonant frequency, and the implied loss factor of the wire. Note that the frequency has been given in $\mathrm{Hz}$.

\begin{tabular}{cccc}
\hline \hline Period $(\mathrm{s})$ & $f_{0}(\mathrm{~Hz})$ & $Q$ & $\phi_{W}$ \\
\hline $4 . \tau$ & $0.2110 \pm 0.0003$ & $83.1 \pm 10$ & $0.0088 \pm 0.0011$ \\
3.4 & $0.2895 \pm 0.0007$ & $168.4 \pm 95$ & $0.0083 \pm 0.0046$ \\
2.6 & $0.3879 \pm 0.0009$ & $290.2 \pm 81$ & $0.0082 \pm 0.0023$ \\
\hline
\end{tabular}





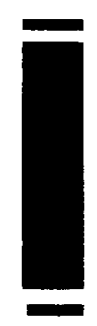

$-$ 